ENTORNOS, No. 27. | Abril 2014

ARTÍCULO DE INVESTIGACIÓN

Recibido: 4 Febrero / Recibido en forma revisada: 5 Marzo / Aceptado: 12 Abril

\title{
Inteligencia emocional como estrategia de afrontamiento frente el Bullying
}

\section{Emotional intelligence as a coping strategy against Bullying}

\section{Emotionale intelligenz als abwehrstrategie gegen Mobbing}

\author{
Juliana Montoya Otálvaro \\ Doctoranda en Psicología Pontificia. Universidad Católica de Argentina. Medellín. Colombia.
} julianaandreamontoyaotalvaro@fumc.edu.co

\section{Resumen}

El objetivo de la presente investigación fue evaluar la inteligencia emocional en estudiantes de secundaria que han sido víctimas, victimarios y espectadores de acoso escolar en una institución educativa en el Municipio de Bello, Antioquia. La investigación se realizó bajo una metodología cuantitativa. Se tomó una muestra de 30 alumnos de secundaria dividido en 2 grupos: 18 de estos fueron reportados por los docentes como víctimas de acoso escolar y 12 reportados como victimarios y espectadores. Se aplicó el CTI, Inventario de pensamiento constructivo. Se concluye que en relación a la inteligencia emocional, en ambos grupos, no se encuentran diferencias significativas, por lo que se puede decir que ambos grupos poseen dificultades en la inteligencia emocional.

Palabras clave: Bullying, inteligencia emocional, estrategias de afrontamiento.

\begin{abstract}
The goal of this research was to assess emotional intelligence in high school students who have been victims, perpetrators and bystanders of bullying in a school in an educational institute in Bello city, Antioquia (Colombia). The research was conducted under a quantitative methodology. 30 Secondary students were chosen as a sample, 18 of these were reported by the teachers as victims of bullying. As a control group, 12 students were chosen in which were found aggressors and viewers. The CTI was applied (Thought Building Inventory). As a conclusion, in realtion to emotional intelligence, in both groups, no significative differences were found, and by that we can say that both groups have difficulties in the emotional intelligence field.
\end{abstract}

Keywords: Bullying, Emotional Intelligence, Coping strategies.

\section{Zusammenfassung}

Das Ziel der vorliegenden Untersuchung war es, die bestehende Verbindung zwischen emotionaler Intelligenz und Mobbing anhand der Schüler der Oberstufe in einer Bildungseinrichtung in Antioquia, Gemeinde Bello, darzustellen. Die Untersuchung wurde mittels der quantitativen Methode durchgeführt. Die Testgruppe bestand aus 30 Schülern der Oberstufe, 18 von ihnen wurden von den Lehrern als Opfer schulischen Mobbings berichtet. Als Kontrollgruppe wurden 12 Schüler ausgewählt, die bereits Täter und Zuschauer waren. Es wurde das Constructive Thinking Inventory (CTI) (Fragebogen zum Konstruktiven Denken) angewandt. Daraus ging hervor, dass bezüglich der emotionalen Intelligenz in beiden Gruppen keine nennenswerten Unterschiede festgestellt wurden, weshalb man sagen kann, dass beide Gruppen eme geringes Maß an emotionaler Intelligenz besaßen.

\section{Introducción}

El bullying o acaso escolar, es un fenómeno que se viene vivenciando en los últimos años en las aulas educativas, en donde las implicaciones no solo van dirigidas a la victima sino que repercute en toda la dinámica educativa en donde se encuentran alumnos, docentes, directivos y padres de familia.

Se podría definir el bullying como una forma de maltrato intencionado y perjudicial de un estudiante hacia otro compañero, generalmente más débil, al que convierte en su víctima recurrente (Olweus 1998)
Palomero y Fernández (2001) definen a su vez el bullying como: "una conducta de persecución y agresión física, psicológica o moral que realiza un alumno o grupo de alumnos sobre otro, con desequilibrio de poder y de manera reiterada". Se puede ver entonces, como la agresión y la búsqueda del poder, son variables significativas en la dinámica de esta problemática.

En relación al contexto colombiano según la ley 1620 de marzo 15 de 2013 de Convivencia escolar, el acoso 
escolar o Bullying es "una conducta negativa, intencional metódica y sistemática de agresión, intimidación, humillación, ridiculización, difamación, coacción, aislamiento deliberado, amenaza o incitación a la violencia o cualquier forma de maltrato psicológico, verbal, físico o por medios electrónicos contra un niño, niña o adolescente, por parte de un estudiante $o$ varios de sus pares con quienes mantiene una relación de poder asimétrica, que se presenta de forma reiterada o a lo largo de un tiempo determinado" (2013, p 20, cítado por Rodríguez, 2013).

Teniendo en cuenta que la violencia hace parte de una de las estrategias que a menudo se utilizan en el acoso escolar, Arón y Milicic (1999, citado por Tamar, 2005) señalan que la violencia siempre apunta a un ejercicio de poder que implica empleo de fuerza, y por lo tanto se da en situaciones de desigualdad y de desequilibrio de poder. Dicha conducta se daría en una "relación social de exceso" en que los límites son transgredidos y se intenta obligar al otro a hacer lo que no haría por su propia voluntad, lo que configura una relación de tipo abusiva.

Loredo, Perea y López (2008) encontraron que todos los actores que hacen parte de este fenómeno comparten ciertas características en común:

1) Exposición crónica a patrones de convivencia violentos: Tanto el agresor como la víctima, viven en ambientes de hostilidad farniliar, escolar, incluso en el área de recreación y deporte.

2) La diversidad de expresión está determinada por las características propias del individuo y de los ambientes (personas y sitios) en los que se desenvuelve.

3) Al final, todos los involucrados, víctimas y agresores, tienen mayor riesgo de sufrir síntomas depresivos e ideación suicida, pasando por trastornos del sueño, enuresis, dolor abdominal, cefalea, malestar general, ansiedad, baja autoestima, sensación de rechazo social, aislamiento, marginación y en general una autopercepción de minusvalía, física, social y hasta económica.

En relación a las consecuencias del acoso escolar, Rigby (2003, citado por Paredes, Álvarez, lega y Vernon, 2008) manifiesta que los resultados de investigaciones transversales sobre el acoso escolar, sugieren que ser continuamente agredido por pares está significativamente relacionado con bajos niveles de bienestar psicológico y ajuste social, con altos niveles de estrés psicológico y con diversos síntomas somáticos. A su vez, los reportes de estudios retrospectivos sugieren que la agresión constante efectuada por pares puede contribuir a dificultades posteriores en salud y bienestar general, mientras que los estudios longitudinales proveen fuerte soporte sobre el hecho de que esta situación es un factor causal significativo de problemas de salud y bienestar emocional, y que los efectos pueden ser duraderos. Más aún, estos estudios indican que la tendencia a victimizar a otros o a otras en la escuela, predice con certeza la conducta antisocial y violenta del adulto.

A raíz del incremento de dicha problemática se han llevado a cabo diversas investigaciones que buscan comprender mejor este fenómeno en contexto colombiano. Chaux, Molano y Podlesky (2009, citado por Hemsohn, Chaux y Molano, 2010) realizaron un estudio con más de 50 mil estudiantes en Colombia, donde encontraron que el $29.1 \%$ de los estudiantes de $5^{\circ}$ y el $14.7 \%$ de los estudiantes de $9^{\circ}$ manifestaron haber sido víctimas de bullying en sus colegios en los últimos dos meses.

En la investigación realizada por Paredes, Alvares, Lega y Vernon (2008) en 14 Colegios de la ciudad de Cali, se encontró la presencia de bullying en el $24.7 \%$ de los 2.542 encuestados, manifestada esta problemática en comportamientos de intimidación o agresión verbal, física y psicológica.

Paredes, Lega, Cabezas, Ortega, Medina y Vega (2011) realizaron un estudio comparativo con agresores y agresoras entre Costa Rica y Colombia, encontrando diferencias en la manifestación de la agresión de acuerdo al género. Los varones costarricenses utilizan más el contacto físico junto a palabras fuertes y la amenaza, mientras que los varones colombianos utilizan más las amenazas, los apodos, la ridiculización y los golpes. Las mujeres costarricenses justifican su agresión en relación con las características de la víctima, mientras que las mujeres colombianas en relación con sus deseos. Se encuentra que el comportamiento de amenaza es generalizado en ambos países y en ambos géneros. Así mismo se estableció en ambos países, la menor utilización de la fuerza física de las mujeres en sus agresiones, lo que podría ser explicado bajo condiciones biológicas.

En un estudio realizado por Cassiani (2013) en 198 estudiantes de sexto a noveno grado de una institución privada de la ciudad de Santiago de Cali, se encontró que la prevalencia del Bullying fue de $20.3 \%$ y así mismo se encontró una asociación significativa a una disfunción familiar leve y severa y a síntomas de ansiedad.

Estas investigaciones evidencian la magnitud del fenómeno del bullying en las aulas colombianas y su impacto en toda la dinámica escolar, por lo tanto al analizar el incremento de esta problemática se hace necesario identificar las diferentes estrategias de afrontamiento que los diferentes implicados están utilizando para el aumento, mantenimiento o disminución del acoso escolar, entendiendo por estrategias de afrontamiento según el modelo de Lazarus y Folkman (1984, citado por Gonzalez, Montoya, Casullo y Bernabéu, 2002) como: "los esfuerzos cognitivos conductuales continuamente cambiantes que realiza el sujeto para manejar las demandas internas $\mathrm{y} / \mathrm{o}$ externas, que son evaluadas como que agotan o exceden los recursos personales". De acuerdo con este modelo las estrategias que se utilizan para afrontar 
una situación estresante depende de la percepción o valoración que el sujeto realiza de la misma en un doble sentido: evaluación primaria, que hace referencia al significado que se le asigna a la situación y evaluación secundaria, a la valoración de las opciones disponibles de afrontamiento. (Mora, 2006).

En la investigación realizada por Mora (2006) se buscaba estudiar los efectos a largo plazo de la participación como víctima en episodios de bullying y de igual forma, analizar el papel medidor que poseen las estrategias de afrontamiento utilizadas para medir el bullying. Se encontró en el estudio, que más que las estrategias de afrontamiento, son las evaluaciones que se hacen del conflicto lo que condicionaran los niveles de estrés en la vida adulta.

López y de la Caba (2011) buscaron en su investigación analizar estrategias de afrontamiento ante el maltrato escolar utilizando una muestra de 1018 estudiantes de 10 a 14 años que cursaban el último ciclo de educación primaria y el primer ciclo de enseñanza secundaria obligatoria. Los resultados arrojaron diferencias estadísticamente significativas entre los cursos, en donde disminuye el peso de estrategias positivas y aumenta el peso de estrategias negativas en los cursos superiores. De igual forma se encontró como respuestas positivas el frenar asertivamente y buscar ayuda y en respuestas negativas se encuentra la reciprocidad negativa (hacer lo mismo o esperar el momento de venganza) y la pasividad.

Elizalde (2010) busco en su estudio describir las estrategias de afrontamiento que utilizan 130 docentes de secundaria de la ciudad de México frente al bullying, encontrando que las más utilizadas son el afrontamiento dirigido al problema, apoyo social, confrontación y revaloración positiva.

Al evaluar las estrategias de afrontamiento en la dinámica del bullying, se identifica la competencia emocional como un elemento fundamental para la intervención de esta problemática por lo que se han desarrollado diversas investigaciones que analizan la relación existente entre la inteligencia emocional y el bullying.

Al hablar de inteligencia emocional esta se refiere a la capacidad de reconocer los propios sentimientos y los ajenos, de motivarse y manejar bien las emociones tanto en relación a uno mismo como a los demás. (Garaigordobil y Oñederra, 2010).

Barón (citado por Ugarriza, 2001) define la inteligencia emocional como un conjunto de habilidades personales, emocionales y sociales y de destrezas que influyen en nuestra habilidad para adaptarnos y enfrentar las demandas y presiones del medio. Como tal, nuestra inteligencia no cognitiva, es un factor importante en la determinación de la habilidad para tener éxito en la vida, influyendo directamente en el bienestar general y en la salud emocional.
En relación al concepto de inteligencia emocional en contexto educativo, Extremera y Fernández (2004) manifiestan que la literatura ha mostrado que las carencias en las habilidades de IE afectan a los estudiantes dentro y fuera del contexto escolar. Los problemas del contexto educativo asociados a bajos niveles de IE serían cuatro:

1. Déficit en los niveles de bienestar y ajuste psicológico del alumnado

2. Disminución en la cantidad y calidad de las relaciones interpersonales

3. Descenso del rendimiento académico

4. Aparición de conductas disruptivas y consumo de sustancias adictivas

Esta carencia de habilidades de IE, puede relacionarse en la forma en que el estudiante afronta la situación de acoso escolar. Salovey (citado por Fernández y Extremera, 2005) manifiesta que los alumnos en el contexto escolar se enfrentan constantemente a situaciones en donde deben recurrir al uso de habilidades emocionales para adaptarse adecuadamente al ambiente escolar. Así mismo, Fernández y Ruiz (2008) expresan que aquellas personas con pocas habilidades emocionales, son más propensas a experimentar estrés y dificultades emocionales durante su proceso académico.

Sánchez, Ortega y Menesini (2012) realizaron un estudio de los diferentes trabajos que analizaban la competencia emocional de los actores del bullying $e$ identificaron tres grandes grupos:

1. Estudio de las habilidades de reconocimiento y atribución emocional

2. Estudio del efecto de las emociones morales y el razonamiento moral juegan en el comportamiento agresivo en general y en el bullying en particular

3. Estudio de las habilidades de regulación emocional y estrategias de afrontamiento ante el estrés en la identificación de los factores relevantes para la explicación y el mantenimiento de la problemática.

Con base a lo anterior y teniendo en cuenta los casos de acoso escolar que se reportaban en la institución donde se llevó a cabo la investigación, se hizo necesario evaluar la inteligencia emocional tanto en victimas como en victimarios y espectadores reportados por los docentes de la Institución y a partir de los resultados diseñar un proyecto de intervención en entrenamiento en inteligencia emocional como estrategia de afrontamiento contra el bullying.

La presente investigación corrobora los resultados arrojados por otras investigaciones que analizan la relación existente entre la inteligencia emocional y el acoso escolar, aportando mayor significado a la competencia emocional en el afrontamiento contra el bullying, así mismo el proyecto de intervención brindado a la Institución donde se realizó la investigación puede ser replicado en diversas instituciones educativas para la prevención e intervención de la problemática. 


\section{Metodología}

La investigación se realizó bajo una metodología cuantitativa mediante una investigación no experimental a través de un diseño transversal de tipo descriptivo.

\section{Instrumento}

CTI. Inventario de pensamiento constructivo. Una medida de la inteligencia emocional. Compuesto por 108 ítems que evalúan el pensamiento constructivo y la inteligencia emocional. El instrumento está jerárquicamente organizado y ofrece información en tres niveles de generalidad. El nivel más general consiste en una escala global que se denomina PCG (Pensamiento Constructivo Global). El siguiente nivel de generalidad lo componen 6 escalas principales que miden las formas básicas de pensamiento constructivo o destructivo que a su vez contienen 15 subes calas: 1) Emotividad: autoestima, tolerancia a la frustración, impasibilidad, capacidad de sobreponerse; 2) Eficacia: pensamiento positivo, actividad, responsabilidad; 3) Pensamiento supersticioso; 4) Rigidez: extremismo, suspicacia, intolerancia; 5) Pensamiento esotérico: creencias paranormales, creencias irracionales; y 6) Ilusión: euforia, pensamiento estereotipado, ingenuidad.

\section{Población y Muestra}

La población fueron estudiantes de secundaria de una institución educativa en el Municipio de Bello, Antioquia. Se realizó un muestreo no probabilístico por conveniencia, puesto que se partió de los casos reportados por los docentes y directivos de la institución. Se tomo una muestra de 30 estudiantes, 12 hombres y 18 mujeres, entre los 11 y 15 años de $6^{a}$ a $9^{a}$ de secundaria. De esta muestra se crearon dos grupos, el primero compuesto por 18 estudiantes reportados como víctimas de acoso escolar y el segundo grupo estuvo conformado por 12 estudiantes reportados como agresores y espectadores.

\section{Análisis de Los Datos}

La corrección del CTI se realizó con un disco de corrección/perfil de los 30 estudiantes cuyos resultados permitieron realizar una discusión en torno a los objetivos de la presente investigación. Posteriormente el análisis de los resultados de dicha corrección se realizo por medio de hoja de datos del paquete estadístico Statgraphics.

\section{Procedimiento}

El interés por realizar esta investigación nace de la necesidad manifestada por la psicóloga y practicantes de la Institución en buscar estrategias de intervención ante los casos de bullying evidenciados dentro de ésta. En un primer momento se revisan los reportes de casos de abuso escolar dentro de la institución recogidos por la psicóloga y los docentes. De los casos reunidos se cuenta con la participación voluntaria de 18 víctimas y 12 victimarios y observadores, se procede a diligenciar el consentimiento informado para adultos responsables de los menores de edad.

Una vez firmados los consentimientos se continúa con la autoaplicación del inventario de pensamiento constructivo. Al tener los resultados de la corrección/perfil de los 30 estudiantes se realiza un análisis estadístico para a partir de allí realizar la discusión de los datos y llegar a conclusiones. A partir de los resultados se diseña un proyecto de intervención en entrenamiento en inteligencia emocional el cual es brindado a la psicóloga de la Institución para llevar a cabo con toda la comunidad educativa.

\section{Resultados}

Para evaluar las puntuaciones totales del cuestionario de pensamiento constructivo aplicado a los 30 estudiantes, se realizó un análisis de cada una de las escalas que evalúa este cuestionario.

\section{Pensamiento Constructivo Global (PCG)}

Esta es una escala global con una medida bipolar ya que integra elementos referentes tanto al pensamiento constructivo como destructivo, incluye elementos de todas las escalas excepto de la Ilusión. En la muestra se observa que tanto en el grupo de victimas como en el grupo control, obtiene un promedio entre 42 y 41 , en donde se puede afirmar que el promedio del PCG no presenta diferencias significativas entre ambos grupos. Según esta puntuación se califica como moderadamente baja la escala de pensamiento constructivo global. Partiendo de esta puntuación se puede afirmar que tanto el grupo de víctimas como el grupo control en la mayoría de las situaciones se le dificulta manejar un pensamiento flexible, se les dificulta aceptar lo que es incontrolable, provocando en ellos una gran angustia y estrés. Poseen una autoestima baja, se les dificulta aceptarse a ellos mismos y a los demás, dudan de los otros y tienden a juzgar negativamente a otros dificultando el establecimiento de relaciones gratificantes.

Gráfica 1. Comparación Pensamiento constructivo global en grupo victimas y no victimas

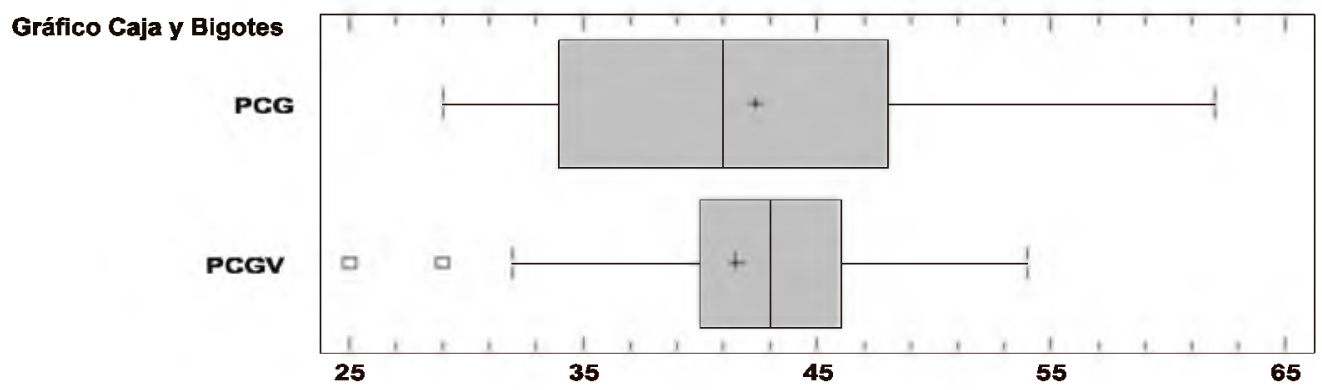


Afrontamiento Emocional (EMO)

Esta es una escala bipolar, más relacionada con la escala global. Quienes obtienen una puntuación alta en esta escala son capaces de enfrentarse a situaciones potencialmente estresantes tomándolas con desafío y no con temor, por lo que experimentan menos estrés ante estas.

Se puede observar que ambos grupos obtienen un promedio de 43 , por lo que se podría afirmar igualmente que el promedio del EMO no presenta diferencias significativas entre ambos grupos. Según esta puntuación se califica como moderadamente baja la escala de afrontamiento emocional.
Partiendo de esta puntuación moderadamente baja se puede afirmar que tanto el grupo de víctimas como el grupo control tienden tomar las cosas de forma personal, son sensibles a las críticas, errores o rechazos. Al recordar adversidades pasadas se sienten afectadas, reaccionan en exceso a las actuales y se preocupan demasiado por las futuras.

Al presentarse ante pensamientos negativos, se les dificulta pensar de forma constructiva como afrontarlos. El afrontamiento emocional se manifiesta en las facetas: Autoestima, tolerancia a la frustración, impasibilidad y capacidad de sobreponerse a experiencias desagradables pasadas, por lo que se puede afirmar que esta muestra presenta dificultades en esta área.

Gráfica 2. Comparacion afrontamiento emocional en grupo víctimas y no víctimas

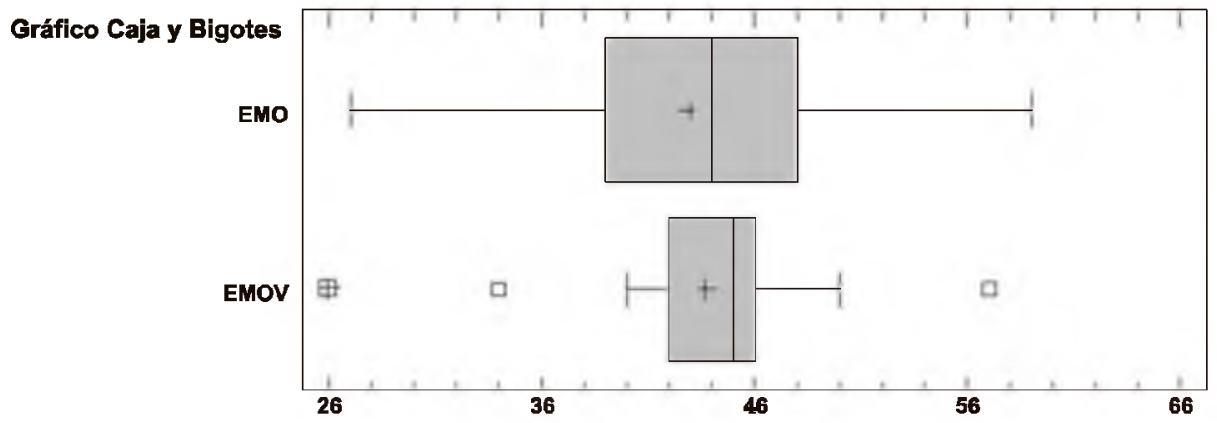

Afrontamiento Conductual (CON)

Constituye una escala bipolar que mide la tendencia a pensar automáticamente en términos que faciliten una acción eficaz. En la muestra se encontró que el grupo de víctimas se encuentra en un rango 25,0 a 61,0 y el grupo control se encuentra en un rango de 37,0 a 63,0. Según esta puntuación se califica como moderadamente baja la escala de afrontamiento conductual en el grupo de víctimas y como moderadamente alta en el grupo control.

Las personas con altas puntuaciones en esta escala son optimistas, entusiastas, energéticos y fiables. Estas personas no son impulsivas, se toman su tiempo para planificar cuando es necesario. Más que juzgar a las personas, juzgan los resultados de formas específicas de comportamiento. Tratan de encontrar soluciones realistas a los problemas. Según las puntuaciones obtenidas están podrian ser características de los estudiantes que pertenecían al grupo de las no víctimas, por lo que se puede afirmar que al puntuar en esta escala moderadamente baja el grupo de víctimas, presentan ciertas dificultas en las facetas que CON evalúa en donde se encuentra el pensamiento positivo, orientación a la acción y responsabilidad.

Estos resultados pueden estar asociados en las características que normalmente presentan aquellas personas que han sido victimas del acoso escolar, en donde se evidencia un poca eficacia al responder a la acción y en donde su pensamiento tiende ser pesimista y polarizado.

Gráfica 3. Comparación afrontamiento conductual en grupo víctimas y no víctimas

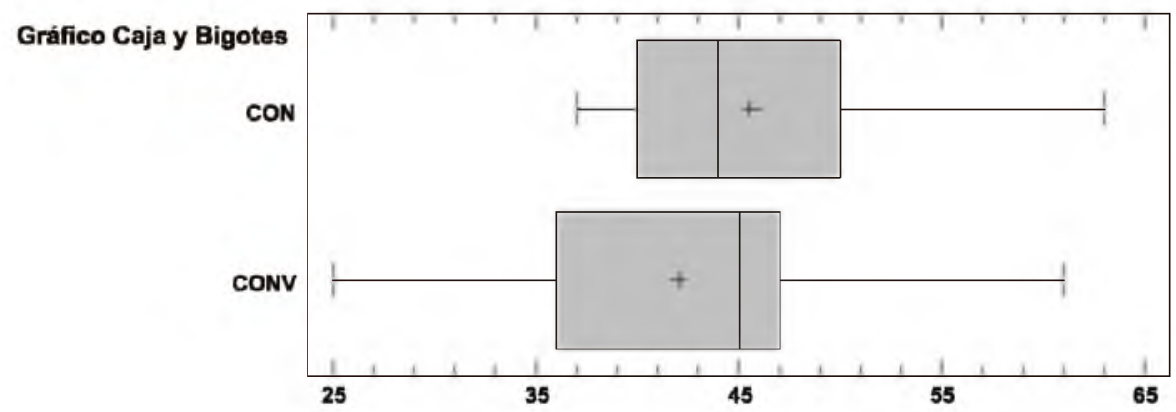




\section{Pensamiento Mágico (MAG)}

Esta escala mide el grado en que las personas se aferran a supersticiones privadas, tales como la creencia que si uno desea mucho una cosa suceda, impedirá que suceda. Según la puntuación de esta escala, en la muestra se encuentra entre los dos grupos un promedio entre 56 y 58, ambos obteniendo puntuaciones moderadamente altas.

Se asocia esta escala fuertemente con el pesimismo, los sentimientos de desesperanza y la depresión. Los sujetos con puntuaciones altas se orientan mas a defenderse de las amenazas que a conseguir la felicidad y la perfección.

Gráfica 4. Comparación Pensamiento mãgico en grupo víctimas y no víctimas

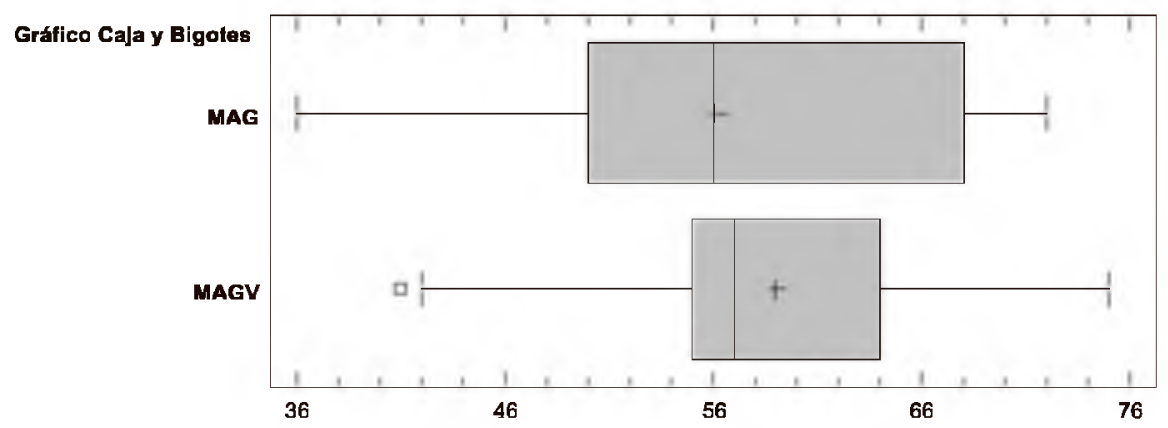

Pensamiento Categórico (CAT)

Esta escala hace referencia a la capacidad de tolerancia y flexibilidad. En las puntuaciones de esta escala se encuentra un promedio entre los dos grupos entre 63 y 65, obteniendo ambos grupos una puntuación moderadamente alta.

Las personas que puntean alto en esta escala son capaces de actuar de forma flexible y reflexiva, acepta los resultados de sus acciones, es capaz de comprender y aceptar el punto de vista de los demás. Las facetas de CAT son: pensamiento polarizado, suspicacia, intransigencia.

En los resultados obtenidos en esta escala, podríamos encontrar incongruencia en relación a las puntuaciones anteriores en donde se encontró dificultades en la eficacia al responder a la acción, estando esto relacionado con una conducta flexible y reflexiva a la cual hace referencia esta escala.

Gráfica 5. Comparación Pensamiento categórico en grupo víctimas y no víctimas

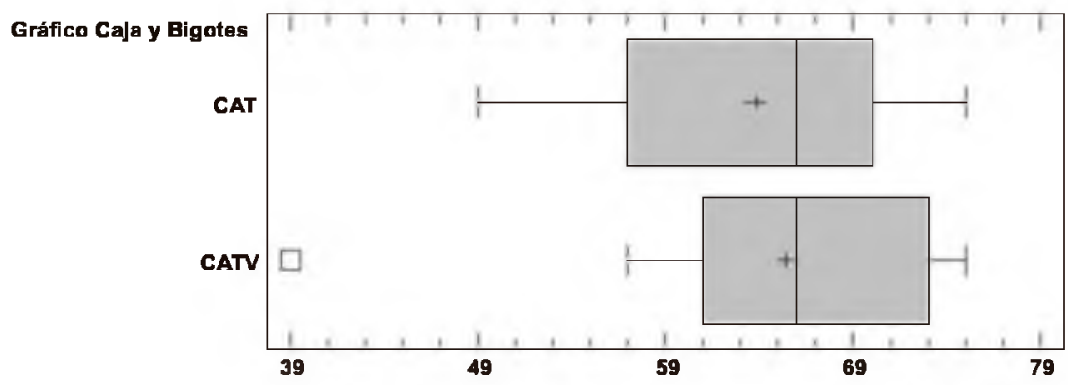

Pensamiento Esotérico (ESO)

Esta escala indica el grado en que la gente cree en fenómenos extraños, mágicos y científicamente cuestionables, como fantasmas, astrología, agueros y las supersticiones convencionales. En lo encontrado en esta escala se observa entre ambos grupos un promedio de 56 y 57 , obteniendo tanto el grupo de víctimas como el grupo de no víctimas una puntuación moderadamente alta.

Puntuaciones muy altas sugieren carencia de pensamiento crítico y una excesiva confianza en las impresiones intuitivas. Las facetas de ESO son creencias paranormales y pensamiento supersticioso.

Generalmente las personas que puntuan alto en esta escala tratan de justificar las consecuencias de sus acciones 
en superticiones, lo que guarda relación en la falta de responsabilidad sobre sus acciones y la dificultad en el pensamiento reflexivo en donde se puntuo bajo en escalas anteriores.

Gráfica 6. Comparación Pensamiento esotérico en grupo víctimas y no víctimas

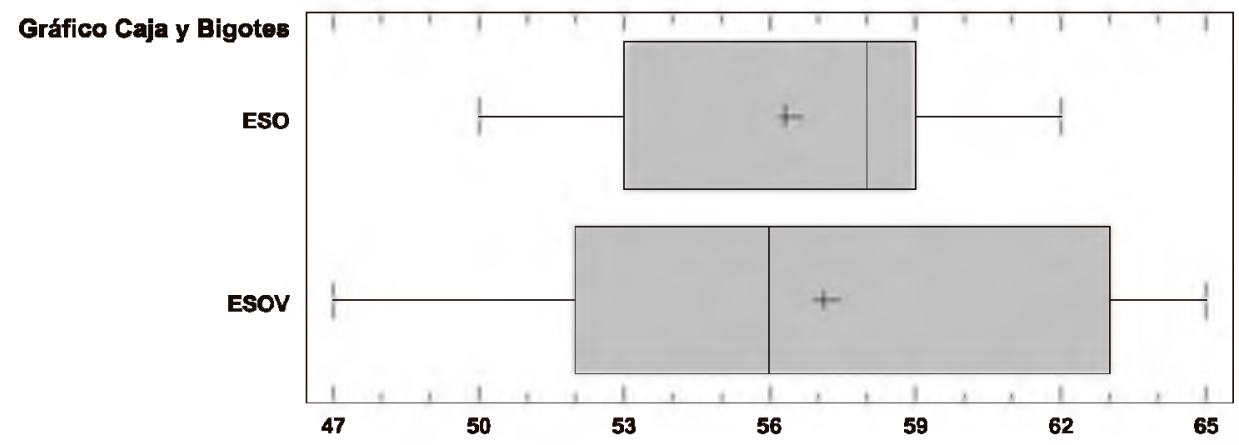

Optimismo Ingenuo (OPT)

Esta escala informa el grado en que una persona es optimista sin fundamento. En la muestra se encuentra un promedio de los dos grupos entre 54 y 56 , puntuando ambos grupos como moderadamente altos.

Si se ve con un enfoque positivo, los optimistas ingenuos son fuertes de espíritu, caen bien a los demás, desde un enfoque negativo, las personas que puntúan alto se asocian a una visión simplista de la vida y una tendencia a evitar realidades desagradables. Las facetas que se tienen en cuenta son optimismo exagerado, pensamiento estereotipado e ingenuidad.

En relación al fenómeno del Bullying, este optimismo ingenuo se puede manifestar en las víctimas en su dificultad de afrontar la situación pensando que las cosas pasaran, que todo será mejor y en muchas ocasiones visto también en los espectadores al no evidenciar el problema al no querer ver realmente la gravedad del asunto, dificultando en muchos casos la oportuna intervención al problema.

Gráfica 7. Comparación Optimismo ingenuo en grupo víctimas y no víctimas

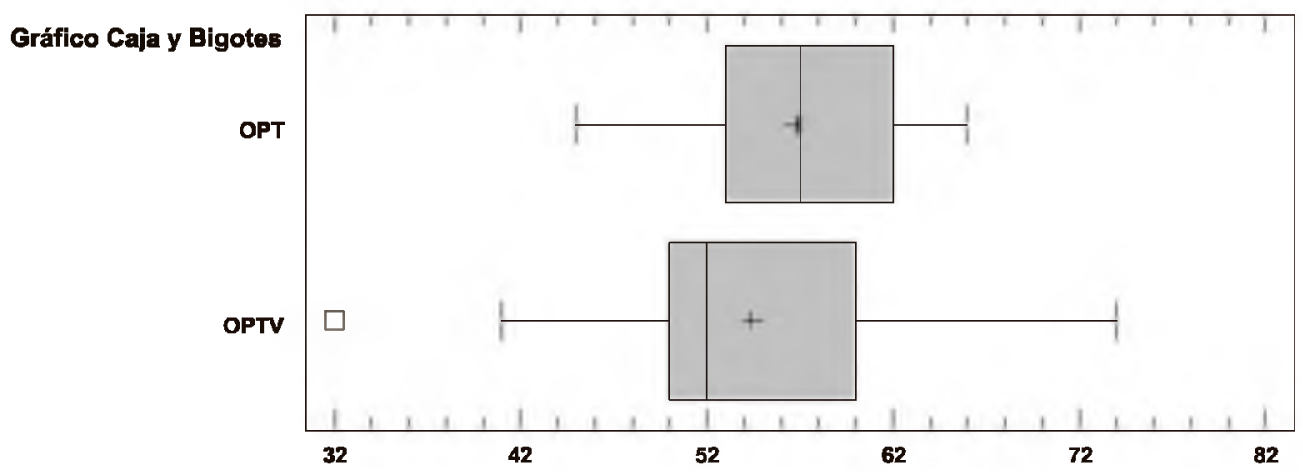

\section{Conclusiones}

A partir de los hallazgos de la investigación se puede concluir que en relación a la inteligencia emocional, tanto el grupo de víctimas como el de no víctimas, en el cual se encontraban victimarios y espectadores, no se encuentran diferencias significativas, por lo que se puede decir que ambos grupos poseen dificultades en la inteligencia emocional, manifestado en el afrontamiento emocional en cuanto a la auto aceptación, la hipersensibilidad y las constantes rumiaciones. Igualmente se evidencia este bajo nivel en inteligencia emocional al analizar las dificultades que se presentan en el afrontamiento conductual en relación a la ausencia o deficiencia de pensamiento positivo, orientación a la acción y la responsabilidad sobre los actos. Así mismo ambos grupos obtienen altas puntuaciones en el pensamiento esotérico y el optimismo ingenuo, siendo estos posibles factores de mantenimiento del fenómeno del bullying, ya que estas características evitan ver la problemática en perspectiva y de esa forma encontrar la adecuada solución. 
Lo aquí encontrado, se evidencia en el estudio realizado por Garaigordobil y Oñederra (2010) tuvo como principal objetivo analizar las relaciones existentes entre ser víctima de acoso escolar y ser agresor con parámetros asociados a la inteligencia emocional (emotividad, eficacia, pensamiento supersticioso, rigidez, pensamiento esotérico, ilusión) en 248 alumnos entre los 12 y 16 años. Los resultados obtenidos arrojaron que los adolescentes que habían sufrido muchas conductas de intimidación, tuvieron bajo nivel de inteligencia emocional, baja emotividad, baja autoestima, baja tolerancia a la frustración, baja eficacia, y poca actividad; y los adolescentes que tenían un nivel alto de conductas antisociales-delictivas mostraban bajo nivel de inteligencia emocional, de eficacia, de actividad, de responsabilidad, y de tolerancia.

Fernández y Extremera (2004) manifiestan:

Desde los modelos de IE también se hace énfasis en las habilidades interpersonales. Las personas emocionalmente inteligentes no sólo serán más hábiles para percibir, comprender y manejar sus propias emociones, sino también serán más capaces de extrapolar sus habilidades de percepción, comprensión y manejo a las emociones de los demás. Esta teoría proporciona un nuevo marco para investigar la adaptación social y emocional puesto que la IE jugaría un papel elemental en el establecimiento, mantenimiento y la calidad de las relaciones interpersonales.

Lo anterior se podría aplicar en el manejo de las emociones tanto de las víctimas como de los mismos agresores dentro del acoso escolar en donde la percepción, la comprensión y el manejo de estas emociones estén constantemente orientadas al establecimiento de adecuadas relaciones interpersonales de cada uno de las personas que conforman la dinámica escolar.

Los hallazgos de la presente investigación, se relaciona con lo encontrado por Collell y Escudé (2004) quienes manifiestan que en general tanto los agresores como las víctimas muestran problemas de regulación emocional y fallan en reconocer los sentimientos y las emociones inducidas por las situaciones de maltrato.

A partir de la revisión realizada por Sánchez, Ortega y Menesini (2012) concluyeron que tanto los agresores como las víctimas del bullying presentan escasa competencia emocional. Los agresores presentan dificultades para adoptar otras perspectivas que no sean las propias $y$ en las victimas las mayores dificultades se presentan en la regulación emocional.

Teniendo en cuenta las consecuencias que el Bullying puede ocasionar, Loredo, Perea y López (2008) manifiestan que independiente de la manera en que son acosadas las víctimas, los resultados tienen impacto en cualquiera de las áreas de su salud. De igual forma, manifiestan que estas consecuencias no solo son esperadas en las victimas sino de igual forma en los observadores y el propio agresor.
Partiendo de lo anterior y basándonos en los resultados de la presente investigación, es de suma importancia en el caso del Bullying el entrenamiento en inteligencia emocional, el cual podría brindar las estrategias de afrontamiento necesarias, en el caso de la victima para enfrentar el rechazo, el aislamiento, el hostigamiento e intimidación e implementar una reacción asertiva ante esta problemática y en el caso del acosador y de los observadores este entrenamiento les brindaría las competencias socioemocionales como la empatía, necesaria para ponerse en el lugar del otro y así frenar la intimidación.

El desarrollo de la IE, por un lado, incrementa el bienestar y la felicidad de las personas y, por otro, les ayuda a afrontar de forma más adaptativa situaciones difíciles incrementando la probabilidad de éxito y minimizando la de fracaso. (Fernández, Berrios, Extremera y Augusto, 2012).

La enseñanza de emociones inteligentes depende de la práctica, el entrenamiento y su perfeccionamiento y no en sí de la instrucción verbal. Lo fundamental es ejercitar y practicar las capacidades emocionales desglosadas en el artículo y convertirlas en una parte más del repertorio emocional del estudiante. De esta forma, técnicas como el modelado y el role-playing emocional se convierten en herramientas básicas de aprendizaje a través de las cuales los educadores, en cuanto "expertos emocionales", plasman su influencia educativa, marcan las relaciones socioafectivas y encauzan el desarrollo emocional de sus alumnos. (Fernández y Extremera, 2002).

En relación a esta educación emocional Buitrón y Navarrete (2008) resaltan la importancia del rol del docente como agente del desarrollo de la inteligencia emocional en los estudiantes, por lo que plantean la necesidad de formar maestros "emocionalmente inteligentes".

Por lo anterior se hace necesario no solo en la intervención sino mucho más importante en la prevención del bullying comenzar a implementar el entrenamiento en inteligencia emocional en las aulas educativas para que por medio de las habilidades socioemocionales tanto de los estudiantes como docentes aprendan a identificar y prevenir las conductas de acoso y así disminuir los índices de este fenómeno que como se vio en la revisión trae consigo consecuencias al desarrollo global de los implicados.

En relación a lo anterior Pepler (2012) expresa que en la intervención del bullying se hace necesario promover las relaciones ya que este no es un problema individual sino que va enfocado a las relaciones por lo que propone un entrenamiento en habilidades sociales que busquen la autorregulación, la regulación emocional, entendimiento moral y empatía.

A partir de los resultados de la presente investigación, se diseñó un proyecto de intervención dirigido al entrenamiento en inteligencia emocional guiado por el 
modelo de habilidad de Mayer y Salovey el cual se centra de forma exclusiva en el procesamiento emocional de la información y en el estudio de las capacidades relacionadas con dicho procesamiento. Desde el modelo de habilidad, la IE implica cuatro grandes componentes: Percepción y expresión emocional, facilitación emocional, comprensión emocional, regulación emocional, a partir de estos se diseñaron 4 talleres que buscan que cada uno de los actores implicados en el fenómeno del bullying adquieran una mayor competencia emocional la cual puedan utilizar como estrategia de afrontamiento ante la problemática. Este proyecto fue entregado a la Institución para ser llevado a cabo por la psicóloga y practicantes de esta, se espera próximamente realizar una evaluación sobre la eficacia del proyecto dentro de esta institución.

\section{Referencias Bibliográficas}

Buitrón \& Navarrete (2008) El docente en el desarrollo de la inteligencia emocional: reflexiones y estrategias. Revista Digital de Investigación en Docencia Universitaria. Año 4 - N 1

Cassiani, C. (2013) Prevalencia de Bullying y factores relacionados en estudiantes de bachillerato de una institución educativa de Cali, Columbia, 2011. 14 $4^{\circ}$ Congreso Virtual de Psiquiatria. Interpsiquis. Recuperado de

http://www.psiquiatria.com/bibliopsiquis/handle/10401/5980

Callcll, J. \& Escudé, C. (2004) Rol de las cmociones cn los procesos de maltrato entre alumnos. Ambits de Psicopedagogia, 12, pp 21-26.

Elizalde, A. (2010) Estudio descriptivo de las estrategias de afrontamiento del bullying en profesorado mexicano. Electronic journal of research in educational psychology. 8(1) 353-372. Recuperado de:

http://www.observatorioperu.com/lecturas\%202010/agosto\%20 2010/Estudio\%20descriptivo\%20de\%20las\%20estrategias\%20de\% 20afrontamiento\%20del\%2 Obullyng,\%20en\%20profesorado\%20m exicano.pdf

Fernández, P. \& Extremera, N. (2002) la inteligencia emocional como una habilidad esencial en la escuela. OEI-Revista Tberoamericana de Educación (ISSN: 1681-5653)

Fernández, P., Herrios, M., Extremera, N. \& Augusto, J (2012) Inteligencia emocional: 22 años de avances empíricos. Behavioral Psychology/Psicología Conductual, Vol. 20, No 1, 2012, pp. 5-13

Fernández, P. \& Extremera, N. (2004). El papel de la inteligencia emocional en el alumnado: evidencias empiricas. Revista Electrónica de Investigación Educativa, 6 Recuperado de: http://redie.uabc.mx/vol6no2/contenido-extremera.html

Fernández, P. \& Extremera, N. (2004) Inteligencia emocional, calidad de las relaciones interpersonales y empatía en estudiantes universitarios. Clínica y salud: Revista de psicología clínica y salud, ISSN $1130-5274$, Vol. 15, No. 2, 2004, págs. 117-137

Fcrnández, P. \& Extremera, N. (2005) La inteligencia emocional y la educación de las emociones desde el modelo de Mayer y Salovey. Revista interuniversitaria de formación del profesorado. No19. Págs. 63-93
Fernández, P. \& Ruiz, d. (2008) Ia inteligencia emocional en la educación. Revista electrónica de Investigación Psicoeducativa. N¹5, Vol. 6. Págs. 421-436

Garaigordobil, M. \& Oñederra, J. 2010. Inteligencia emocional en las víctimas de acoso escolar y en los agresores. Furopean Journal of Education and Psychology, Vol. 3, N 2 Págs. 243-256

González, R; Montoya, Ii Casullo, M. \& Bernabéu, J. (2002) Relación entre estilos $y$ estrategias de afrontamiento y bienestar psicológico en adolescentes. Psicothema. Vol. $14, n^{\circ} 2$, pp. 363 368 .

Heinsohn R- Chaux F; \& Molano A (2010) "La chispita que quería encender todos los fósforos": Percepciones, creencias y emociones frente a la intimidación en un colegio masculino. Voces y Silencios: Revista Latinoamericana de Educación, Vol. 1, No. 1, 5-22.

López, R. \& De la Caba, M. (2011) Estrategias de afrontamiento ante el maltrato cscolar en estudiantes de primaria y secundaria. Aula abierta. Vol. $39 \mathrm{~N}^{\circ} 1$. PP. 59-68

Loredo, A., Perea, A. \& López, G. (2008) "Bullying": acoso escolar. La violencia entre iguales. Problemática real en adolescentes. Acta Pediátrica México; 29(4):210-4

Mora, J (2006) Las estrategias de afrontamiento ¿Medidoras de los efectos a largo plazo de las víctimas del bullying? Anuario de psicología clínica y de la salud. Vol. 2 Págs. 15-26

Olweus, Dan (1998). Conductas de acoso y amenaza entre escolares. Madrid: Ediciones Morata.

Palomero. \& Fernández, M. (2001) La violencia Escolar: un punto de vista global. Revista interuniversitaria de formación del profesorado. $\mathrm{N} \quad \mathbf{4 1}$. Págs. 18-38

Paredes, M., Álvarez, M. Lega, L. \& Vernon, A. (2008). Estudio exploratorio sobre el fenómeno del "bullying" en la ciudad de Cali, Colombia. Revista Latino Americana de Ciencias Sociales en Niñez y Juventud, 6 (1), pp. 295-317.

Paredes, M; Lega, I; Cabezas, H; Ortega, M; Medina, Y; \& Vega, C. (2011). Diferencias Transculturales en la Manifestación del Bullying en Estudiantes de Escucla Sccundaria. Revista Latinoamcricana de Ciencias Sociales, Nin̄ez y Juventud, 2 (9), pp. 761 - 768.

Pepler, D. (2012) Healthy development depends on healthy relationships. II Simposio internacional de Bullying. Universidad CES Medellín.

Pérez, G. (2004) Dinámica bullying y psicopatología en adolescentes. $5^{\circ}$ Congreso virtual de Psiquiatría. http://www.psiquiatria.com/bibliopsiquis/bitstream/10401/31 73/1/interpsiquis_2004_13481.pdf

Proyecto de Ley No. 064 de (2009). Acoso escolar dispara las alarmas en Colombia Disponible en: http://profamilia.com/index.php?option=com_content\&view= article\&id=534:acoso-escolar-dispara-las-alarmas-en-colombia

Rodriguez, R. (2013) Ensayo argumentativo "el bullying". Recuperado de: http://repository.unimilitar.edu.co/bitstream/10654/11251 /1/RodriguezBejaranoRosa2013.pdf

Sánchez, V; Ortega, R. \& Menesini, E. (2012) La competencia emocional de agresores y víctimas de bullying. Anales de psicología. Vol. 28 No1. Pags 71-82

Tamar, F. (2005) Maltrato Entre Escolares (Bullying): Estrategias de Manejo que Implementan los Profesores al Interior del Establecimiento Escolar. Psykhe v. 14 n. 1

Ugarriza. (2001) La evaluación de la inteligencia emocional a través del inventario de Barón (I-CE) en una muestra de Lima Metropolitana. Recuperado dc:

http://redalyc. uaemex.mx/src/inicio/ArtPdfRed.jsp?iCve=14711 8178005. ISSN 1560-6139 